Research Article

\title{
Exact Artificial Boundary Conditions for Quasi-Linear Problems in Semi-Infinite Strips
}

\author{
Yajun Chen $\mathbb{D}^{1,2}$ and Qikui Du ${ }^{1}$ \\ ${ }^{1}$ School of Mathematical Sciences, Nanjing Normal University, Nanjing 210023, China \\ ${ }^{2}$ Department of Mathematics, Shanghai Maritime University, Shanghai 200136, China
}

Correspondence should be addressed to Yajun Chen; chenyajun@shmtu.edu.cn

Received 1 June 2021; Accepted 30 August 2021; Published 21 September 2021

Academic Editor: Xian-Ming Gu

Copyright (c) 2021 Yajun Chen and Qikui Du. This is an open access article distributed under the Creative Commons Attribution License, which permits unrestricted use, distribution, and reproduction in any medium, provided the original work is properly cited.

In this paper, the exact artificial boundary conditions for quasi-linear problems in semi-infinite strips are investigated. Based on the Kirchhoff transformation, the exact and approximate boundary conditions on a segment artificial boundary are derived. The error estimate for the finite element approximation with the artificial boundary condition is obtained. Some numerical examples show the efficiency of this method.

\section{Introduction}

The quasi-linear problems in semi-infinite strips have many physical applications in the field of magnetostatics or compressible flow around an obstacle in a channel. There have been many relevant works about quasi-linear problems in bounded domains, for example, the Galerkin approximations $[1,2]$, the finite element method $[3,4]$, and the mixed finite element method [5-7] for quasi-linear problems. One can refer to [8-10], for more related works.

The artificial boundary method $[11,12]$, which is also called coupling of the finite element method with natural boundary reduction [13-15] or the DtN method [16, 17], is a common method to deal with quasi-linear problems in unbounded domains. In the last decade, artificial boundaries of various shapes have been derived for quasi-linear problems in unbounded domains. Circular $[18,19]$ and elliptical [20] artificial boundaries are for two-dimensional problems, spheroidal artificial boundaries [18] are for three-dimensional problems, and circular arc artificial boundaries [21] are for problems in concave angle domains.

The purpose of this paper is to propose an artificial boundary method of using a segment artificial boundary for quasi-linear problems in semi-infinite strips. The segment artificial boundary we proposed in this paper is different with the circular artificial boundary in [18]. We also obtain an error estimate in Section 3, which was not discussed in [18].

Let $\Omega$ be a strip, and $b$ is the width of the channel $\Omega$. The boundary of domain $\Omega$ is decomposed into three disjoint parts: $\Gamma_{W}, \Gamma_{N}$, and $\Gamma_{S}$ (see Figure 1). We introduce a Cartesian coordinate system $\left(x_{1}, x_{2}\right)$, such that the ray $\Gamma_{S}$ coincides with the $x_{1}$-axis.

We consider the following quasi-linear problem:

$$
\left\{\begin{array}{l}
-\left(\frac{\partial}{\partial x_{1}}\left(a(x, u) \frac{\partial u}{\partial x_{1}}\right)+\frac{\partial}{\partial x_{2}}\left(a(x, u) \frac{\partial u}{\partial x_{2}}\right)\right)=f, \quad \text { in } \Omega, \\
\frac{\partial u}{\partial n}=0, \quad \text { on } \Gamma_{N} \mathrm{U}_{S}, \\
u=0, \quad \text { on } \Gamma_{W}, \\
u(x) \text { is bounded, as } x_{1} \longrightarrow+\infty,
\end{array}\right.
$$

where $n$ is the unit exterior normal vector on $\Gamma_{N}$ or $\Gamma_{S}$ and $a(x, u)$ and $f$ are two given functions.

Suppose that $\partial a / \partial s$ and $\partial^{2} a / \partial s^{2}$ are continuous, and $a(\cdot, \cdot)$ satisfies $[1]$ 


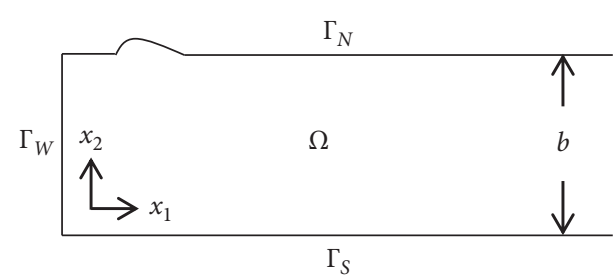

Figure 1: The illustration of domain $\Omega$.

$0<C_{0} \leq a(x, u) \leq C_{1}, \quad \forall u \in \mathbb{R}$, and for almost all $x \in \Omega$,

where $C_{0}$ and $C_{1}$ are two positive constants, and

$$
\begin{aligned}
& |a(x, u)-a(x, v)| \leq C_{L}|u-v|, \\
& \forall u, v \in \mathbb{R} \text {, and for almost all } x \in \Omega,
\end{aligned}
$$

where $C_{L}>0$ is a positive constant. We also assume that $f \in L^{2}(\Omega)$ has compact support, i.e., there exists a constant $d_{0}>0$, such that

$$
\operatorname{supp} f \subset \Omega_{d_{0}}=\left\{x \in \Omega \mid x_{1} \leq d_{0}\right\} .
$$

Additionally, we suppose that

$$
a(x, u) \equiv \widetilde{a}(u), \quad \text { when } x_{1} \geq d_{0} .
$$

The rest of the paper is organized as follows. In Section 2, we derive the exact artificial boundary condition on a segment. In Section 3, we discuss the finite element approximation and a new error estimate. In Section 4, we give some numerical examples to show the efficiency of the method. The conclusions are given in Section 5.

\section{Exact Quasi-Linear Artificial Boundary Condition}

We introduce a segment artificial boundary $\Gamma_{E}=\left\{\left(x_{1}\right.\right.$, $\left.\left.x_{2}\right) \mid x_{1}=d, \quad 0 \leq x_{2} \leq b\right\}$ to enclose supp $f$, which divides $\Omega$ into a bounded domain $\Omega_{W}$ and an unbounded domain $\Omega_{E}$ (see Figure 2).

Then, the original problem (1) can be described in the coupled form:

$$
\begin{aligned}
& \left\{\begin{array}{l}
-\left(\frac{\partial}{\partial x_{1}}\left(a(x, u) \frac{\partial u}{\partial x_{1}}\right)+\frac{\partial}{\partial x_{2}}\left(a(x, u) \frac{\partial u}{\partial x_{2}}\right)\right)=f, \text { in } \Omega_{W} \\
\frac{\partial u}{\partial n}=0, \text { on } \Gamma_{N W} U \Gamma_{S W}, \\
u=0, \text { on } \Gamma_{W},
\end{array}\right. \\
& \left\{\begin{array}{l}
-\left(\frac{\partial}{\partial x_{1}}\left(\tilde{a}(u) \frac{\partial u}{\partial x_{1}}\right)+\frac{\partial}{\partial x_{2}}\left(\tilde{a}(u) \frac{\partial u}{\partial x_{2}}\right)\right)=0, \text { in } \Omega_{E}, \\
\frac{\partial u}{\partial n}=0, \text { on } \Gamma_{N E} U \Gamma_{S E}, \\
u(x) \text { is bounded, as } x_{1} \longrightarrow+\infty,
\end{array}\right.
\end{aligned}
$$

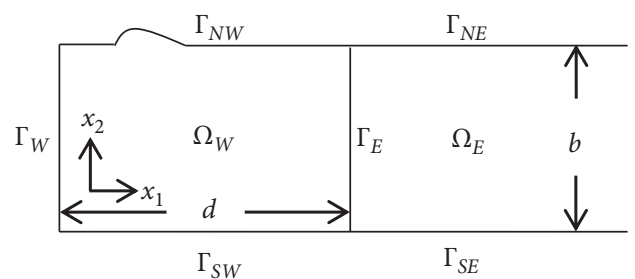

Figure 2: The illustration of domain $\Omega_{W}$ and $\Omega_{E}$.

$$
u(x) \text { and } \widetilde{a}(u) \frac{\partial u}{\partial n}
$$

$$
\text { are continuous on the artificial boundary } \Gamma_{E} \text {, }
$$

where $\Gamma_{N W}=\Gamma_{N} \cap \Omega_{W}, \Gamma_{S W}=\Gamma_{S} \cap \Omega_{W}, \Gamma_{N E}=\Gamma_{N} \cap \Omega_{E}$, and $\Gamma_{S E}=\Gamma_{S} \cap \Omega_{E}$.

We introduce the Kirchhoff transformation [22]:

$$
w(x)=\int_{0}^{u(x)} \tilde{a}(\xi) d \xi, \text { for } x \in \Omega_{E} .
$$

Since $\tilde{a}(u)$ is a positive function, transformation (9) is invertible. Notice that

$$
\nabla w=\widetilde{a}(u) \nabla u .
$$

Then, quasi-linear problem (1) can be transformed into a linear problem as follows:

$$
\left\{\begin{array}{l}
-\Delta w=0, \quad \text { in } \Omega_{E}, \\
\frac{\partial w}{\partial n}=0, \quad \text { on } \Gamma_{N E} \mathrm{U} \Gamma_{S E}, \\
w(x) \text { is bounded, as } x_{1} \longrightarrow+\infty .
\end{array}\right.
$$

By the natural boundary reduction [13-15], we know that the solution of problem (11) has the Fourier series expansion:

$$
w\left(x_{1}, x_{2}\right)=\frac{a_{0}}{2}+\sum_{n=1}^{+\infty} a_{n} e^{\left(d-x_{1}\right)(n \pi / b)} \cos \frac{n \pi x_{2}}{b},
$$

where

$$
a_{n}=\frac{2}{b} \int_{0}^{b} w\left(d, x_{2}^{\prime}\right) \cos \frac{n \pi x_{2}^{\prime}}{b} \mathrm{~d} x_{2}^{\prime}, \quad n=0,1,2, \cdots .
$$
obtain

We differentiate (12) with respect to $x_{1}$ and set $x_{1}=d$ to

$$
\left.\frac{\partial w}{\partial x_{1}}\left(x_{1}, x_{2}\right)\right|_{x_{1}=d}=-\frac{2 \pi}{b^{2}} \sum_{n=1}^{+\infty} n \int_{0}^{b} w\left(d, x_{2}^{\prime}\right) \cos \frac{n \pi x_{2}^{\prime}}{b} \cos \frac{n \pi x_{2}}{b} \mathrm{~d} x_{2}^{\prime}
$$

Since

$$
\widetilde{a}(u) \frac{\partial u}{\partial n}=\frac{\partial w}{\partial n}=-\frac{\partial w}{\partial x_{1}},
$$

we have the exact artificial boundary condition of $u$ on $\Gamma_{E}$ : 


$$
\begin{gathered}
\widetilde{a}(u) \frac{\partial u}{\partial n}=\frac{2 \pi}{b^{2}} \sum_{n=1}^{+\infty} n \int_{0}^{b}\left(\int_{0}^{u\left(d, x_{2}^{\prime}\right)} \tilde{a}(\xi) \mathrm{d} \xi\right) \\
\cos \frac{n \pi x_{2}^{\prime}}{b} \cos \frac{n \pi x_{2}}{b} \mathrm{~d} x_{2}^{\prime} \triangleq \mathscr{K} u\left(d, x_{2}\right) .
\end{gathered}
$$

By the exact artificial boundary condition (16), we obtain

$$
\left\{\begin{array}{l}
-\left(\frac{\partial}{\partial x_{1}}\left(a(x, u) \frac{\partial u}{\partial x_{1}}\right)+\frac{\partial}{\partial x_{2}}\left(a(x, u) \frac{\partial u}{\partial x_{2}}\right)\right)=f, \in \text { in } \Omega_{W} \\
\frac{\partial u}{\partial n}=0, \text { on } \Gamma_{N W} \mathrm{U} \Gamma_{S W}, \\
u=0, \text { on } \Gamma_{W}, \\
\tilde{a}(u) \frac{\partial u}{\partial n}=\mathscr{K} u\left(d, x_{2}\right), \text { on } \Gamma_{E} .
\end{array}\right.
$$

Suppose $V=\left\{v \in H^{1}\left(\Omega_{W}\right)|v|_{\Gamma_{W}}=0\right\}$; then, problem (17) is equivalent to the following variational problem:

$$
\left\{\begin{array}{l}
\text { Find } u \in V, \quad \text { such that, } \\
A(u ; u, v)+B(u ; u, v)=F(v), \quad \forall v \in V,
\end{array}\right.
$$

where

$$
\begin{aligned}
& A(w ; u, v)=\int_{\Omega_{W}} a(x, w)\left(\frac{\partial u}{\partial x_{1}} \frac{\partial v}{\partial x_{1}}+\frac{\partial u}{\partial x_{2}} \frac{\partial v}{\partial x_{2}}\right) \mathrm{d} x \\
& B(w ; u, v)=\sum_{n=1}^{+\infty} \frac{2}{n \pi} \int_{0}^{b} \int_{0}^{b} \tilde{a}\left(w\left(d, x_{2}^{\prime}\right)\right) \frac{\partial u}{\partial x_{2}^{\prime}} \\
& \cdot\left(d, x_{2}^{\prime}\right) \frac{\partial v}{\partial x_{2}}\left(d, x_{2}\right) \sin \frac{n \pi x_{2}^{\prime}}{b} \sin \frac{n \pi x_{2}}{b} \mathrm{~d} x_{2}^{\prime} \mathrm{d} x_{2}, \\
& F(v)=\int_{\Omega_{W}} f(x) v(x) \mathrm{d} x . \\
& \forall H^{s}\left(\Gamma_{E}\right) \Leftrightarrow v\left(d, x_{2}\right)=\frac{c_{0}}{2}+\sum_{n=1}^{+\infty} c_{n} \cos \frac{n \pi x_{2}}{b} \text { and } \frac{c_{0}^{2}}{2} \\
& +\sum_{n=1}^{+\infty}\left(1+n^{2}\right)^{s} c_{n}^{2}<\infty
\end{aligned}
$$

The norm of $H^{s}\left(\Gamma_{E}\right)$ can be defined as follows:

$$
\left\|v\left(d, x_{2}\right)\right\|_{s, \Gamma_{E}}=\left[\frac{c_{0}^{2}}{2}+\sum_{n=1}^{+\infty}\left(1+n^{2}\right)^{s} c_{n}^{2}\right]^{1 / 2} .
$$

Then, we obtain the following lemma.
Lemma 1. The bilinear form $B(u ; u, v)$ is symmetric, continuous, and semidefinite on $V \times V$.

Proof. For $u, v \in V$, we suppose that

$$
\begin{aligned}
& u\left(d, x_{2}^{\prime}\right)=\frac{a_{0}}{2}+\sum_{n=1}^{+\infty} a_{n} \cos \frac{n \pi x_{2}^{\prime}}{b} \\
& v\left(d, x_{2}\right)=\frac{c_{0}}{2}+\sum_{n=1}^{+\infty} c_{n} \cos \frac{n \pi x_{2}^{\prime}}{b} .
\end{aligned}
$$

Taking the derivative with respect to $x_{2}^{\prime}$ and $x_{2}$, we obtain

$$
\begin{aligned}
& \frac{\partial u}{\partial x_{2}^{\prime}}\left(d, x_{2}^{\prime}\right)=\sum_{n=1}^{+\infty} \frac{n \pi}{b} a_{n} \sin \frac{n \pi x_{2}^{\prime}}{b}, \\
& \frac{\partial v}{\partial x_{2}}\left(d, x_{2}\right)=\sum_{n=1}^{+\infty} \frac{n \pi}{b} c_{n} \sin \frac{n \pi x_{2}}{b} .
\end{aligned}
$$

Then, we have

$$
\begin{aligned}
|B(u ; u, v)| & \leq C\left(\sum_{n=1}^{+\infty} n a_{n}^{2}\right)^{1 / 2}\left(\sum_{n=1}^{+\infty} n c_{n}^{2}\right)^{1 / 2} \\
& \leq C\|u\|_{1 / 2, \Gamma_{E}}\|v\|_{1 / 2, \Gamma_{E}} \leq C\|u\|_{1, \Omega_{W}}\|v\|_{1, \Omega_{W}} .
\end{aligned}
$$

Next, we show that $B(u ; u, v)$ is semidefinite. For any given $v \in V$, we consider the auxiliary problem as follows:

$$
\left\{\begin{array}{l}
-\left(\frac{\partial}{\partial x_{1}}\left(a(x, u) \frac{\partial u}{\partial x_{1}}\right)+\frac{\partial}{\partial x_{2}}\left(a(x, u) \frac{\partial u}{\partial x_{2}}\right)\right)=0, \text { in } \Omega_{E} \\
\frac{\partial u}{\partial n}=0, \text { on } \Gamma_{N E} U \Gamma_{S E}, \\
u=v, \text { on } \Gamma_{E} \\
u(x) \text { is bounded, as } x_{1} \longrightarrow+\infty
\end{array}\right.
$$

The solution $u$ of the above problem satisfies

$$
\tilde{a}(u) \frac{\partial u}{\partial n}=\mathscr{K} u\left(d, x_{2}\right)
$$

We multiply (27) by $u$ and integrate over $\Omega_{E}$; then, we have

$$
B(u ; u, u)=\int_{\Omega_{E}} \tilde{a}(u)|\nabla u|^{2} \mathrm{~d} x \geq 0 .
$$

This completes the proof.

In practice, we have to truncate the infinite series in (16) by finite terms; let

$$
\mathscr{K}^{N} u=\frac{2 \pi}{b^{2}} \sum_{n=1}^{N} n \int_{0}^{b}\left(\int_{0}^{u\left(d, x_{2}^{\prime}\right)} \tilde{a}(\xi) d \xi\right) \cos \frac{n \pi x_{2}^{\prime}}{b} \cos \frac{n \pi x_{2}}{b} \mathrm{~d} x_{2}^{\prime} .
$$

Consider the approximation problem 


$$
\left\{\begin{array}{l}
-\left(\frac{\partial}{\partial x_{1}}\left(a\left(x, u^{N}\right) \frac{\partial u^{N}}{\partial x_{1}}\right)+\frac{\partial}{\partial x_{2}}\left(a\left(x, u^{N}\right) \frac{\partial u^{N}}{\partial x_{2}}\right)\right)=f, \text { in } \Omega_{W}, \\
\frac{\partial u^{N}}{\partial n}=0, \quad \text { on } \Gamma_{N W} \mathrm{U}_{S W}, \\
u^{N}=0, \quad \text { on } \Gamma_{W}, \\
\tilde{a}\left(u^{N}\right) \frac{\partial u^{N}}{\partial n}=\mathscr{K}^{N} u^{N}, \quad \text { on } \Gamma_{E} .
\end{array}\right.
$$

Problem (31) is equivalent to the following variational problem:

$$
\left\{\begin{array}{l}
\text { Find } u^{N} \in V, \text { such that, } \\
A\left(u^{N} ; u^{N}, v\right)+B_{N}\left(u^{N} ; u^{N}, v\right)=F(v), \quad \forall v \in V,
\end{array}\right.
$$

where

$$
\begin{aligned}
B_{N}(w ; u, v)= & \sum_{n=1}^{N} \frac{2}{n \pi} \int_{0}^{b} \int_{0}^{b} \widetilde{a}\left(w\left(d, x_{2}^{\prime}\right)\right) \\
& \frac{\partial u}{\partial x_{2}^{\prime}}\left(d, x_{2}^{\prime}\right) \frac{\partial v}{\partial x_{2}}\left(d, x_{2}\right) \sin \frac{n \pi x_{2}^{\prime}}{b} \sin \\
& \frac{n \pi x_{2}}{b} \mathrm{~d} x_{2}^{\prime} \mathrm{d} x_{2} .
\end{aligned}
$$

Similar with Lemma 1 , we have

Lemma 2. The bilinear form $B_{N}(u ; u, v)$ is symmetric, continuous, and semidefinite on $V \times V$.

\section{Finite Element Approximation}

Suppose that $\mathscr{J}_{h}$ is a quasi-uniform and regular triangulation of $\Omega_{W}$ such that

$$
\Omega_{W}=U_{K \in \mathscr{F}_{h}} K,
$$

where $K$ is a (curved) triangle and $h$ is the maximal diameter of the triangles. Let

$$
V_{h}=\left\{v_{h} \in V, v_{h} \mid K \text { is a linear polynomial, } \quad \forall K \in \mathscr{F}_{h}\right\} .
$$

We consider the approximation problem of (32):

$$
\left\{\begin{array}{l}
\text { Find } u_{h}^{N} \in V_{h} \text {, such that } \\
A\left(u_{h}^{N} ; u_{h}^{N}, v_{h}\right)+B_{N}\left(u_{h}^{N} ; u_{h}^{N}, v_{h}\right)=F\left(v_{h}\right), \quad \forall v_{h} \in V_{h} .
\end{array}\right.
$$

Theorem 1. The variational problems (18), (32), and (36) are uniquely solvable.

Proof. By (2), we have

$$
\begin{aligned}
& |A(u ; v, v)| \geq C_{0}\|v\|_{1, \Omega_{W}}^{2}, \\
& |A(u ; u, v)| \leq C_{1}\|u\|_{1, \Omega_{W}}\|v\|_{1, \Omega_{W}} .
\end{aligned}
$$

This means that $A(u ; u, v)$ is coercive and bounded in $V$. From Lemma 1 , we obtain that $A(u ; u, v)+B(u ; u, v)$ is also coercive and bounded in $V$. By (3), we get that $a(x, u)$ is uniformly Lipschitz continuous with respect to $u$. Under these conditions, referring to [1], we obtain that variational problem (18) has a unique solution $u \in V$, for all $f \in L^{2}(\Omega)$. It is easy to deduce that problems (32) and (36) are uniquely solvable in the same way.

We assume $u, u^{N} \in H^{2}\left(\Omega_{W}\right)$ and $u_{h}^{N} \in V_{h}$ are the solutions of problems (18), (32), and (36), respectively. We also suppose that

$$
V_{h} \subset V \cap W^{1,2+\varepsilon}\left(\Omega_{W}\right) \text { for some } \varepsilon \in(0,1) .
$$

Additionally, we require that $\left\{V_{h}\right\}_{h} \longrightarrow 0$ is a family of finite-dimensional subspaces of $V \cap C\left(\Omega_{W}\right)$, such that, for any

$$
\begin{aligned}
& v \in V \cap C\left(\Omega_{W}\right), \text { there exists }\left\{v_{h}\right\}: v_{h} \in V_{h}, \\
& \left\|v-v_{h}\right\| \longrightarrow 0, \text { as } h \longrightarrow 0, \\
& \left\|v_{h}\right\|_{1,2+\varepsilon, \Omega_{W}} \leq C(v), \text { for any } h,
\end{aligned}
$$

where $C(v)>0$ is independent of $h$.

Then, we obtain that the continuous piecewise polynomial spaces (35) satisfy condition (38). Moreover, if we assume $v_{h}=\Pi_{h} v$, where $\Pi_{h}: v \longrightarrow v_{h}$ is the interpolation operator, then, by (40), we obtain

$$
\left\|v_{h}\right\|_{1,2+\varepsilon, \Omega_{W}} \leq\left\|\Pi_{h} v-v\right\|_{1,2+\varepsilon, \Omega_{W}}+\|v\|_{1,2+\varepsilon, \Omega_{W}} \leq C(v) .
$$

Following the convergence theory in $[4,15]$, we have the result as follows:

$\lim _{h \longrightarrow 0}\left\|u_{h}^{N}-u^{N}\right\|_{1, \Omega_{W}}=0$ and $u^{N} \subset V \cap W^{1,2+\varepsilon}\left(\Omega_{W}\right), \forall N \geq 0$.

Furthermore, we have the following lemma.

Lemma 3. Suppose $u$ is the solution of (18) and $u^{N}$ is the solution of (32); we have

$$
\lim _{N \longrightarrow+\infty}\left\|u-u^{N}\right\|_{1, \Omega_{W}}=0 .
$$

Proof. From (2) and Theorem 2, we have

$$
\begin{aligned}
\left\|u^{N}\right\|_{1, \Omega_{W}}^{2} \leq & C\left(A\left(u^{N} ; u^{N}, u^{N}\right)+B\left(u^{N} ; u^{N}, u^{N}\right)\right) \\
= & C\left(F\left(u^{N}\right)+B\left(u^{N} ; u^{N}, u^{N}\right)-B_{N}\left(u^{N} ; u^{N}, u^{N}\right)\right) \\
\leq & C\left(\|f\|_{0, \Omega_{W}}\|u\|^{N}{ }_{1, \Omega_{W}}+\mid B\left(u^{N} ; u^{N}, u^{N}\right)\right. \\
& \left.-B_{N}\left(u^{N} ; u^{N}, u^{N}\right) \mid\right) .
\end{aligned}
$$

For $u^{N} \in V$, we suppose 


$$
\begin{aligned}
w^{N}\left(x_{1}, x_{2}^{\prime}\right) & =\int_{0}^{u^{N}\left(x_{1}, x_{2}^{\prime}\right)} \tilde{a}(\xi) d \xi \\
& =\frac{a_{0}}{2}+\sum_{n=1}^{+\infty} a_{n} e^{\left(d_{0}-x_{1}\right)(n \pi / b)} \cos \frac{n \pi x_{2}^{\prime}}{b}, \quad \forall x_{1}>d_{0}, \\
u^{N}\left(d, x_{2}\right) & =\frac{c_{0}}{2}+\sum_{n=1}^{+\infty} c_{n} \cos \frac{n \pi x_{2}}{b} .
\end{aligned}
$$

Then,

$$
\begin{aligned}
& \left|B\left(u^{N} ; u^{N}, u^{N}\right)-B_{N}\left(u^{N} ; u^{N}, u^{N}\right)\right| \\
& =\left|\sum_{n=N+1}^{+\infty} \frac{2}{n \pi} \int_{0}^{b} \int_{0}^{b} \frac{\partial w^{N}}{\partial x_{2}^{\prime}}\left(d, x_{2}^{\prime}\right) \frac{\partial u^{N}}{\partial x_{2}}\left(d, x_{2}\right) \sin \frac{n \pi x_{2}^{\prime}}{b} \sin \frac{n \pi x_{2}}{b} \mathrm{~d} x_{2}^{\prime} \mathrm{d} x_{2}\right| \\
& =\left|\sum_{n=N+1}^{+\infty} \frac{n \pi}{2} e^{\left(d_{0}-d\right)(n \pi / b)} a_{n} c_{n}\right| \leq C e^{\left(d_{0}-d\right)(((N+1) \pi) / b)}\left(\sum_{n=N+1}^{+\infty} n a_{n}^{2}\right)^{1 / 2}\left(\sum_{n=N+1}^{\infty} n c_{n}^{2}\right)^{1 / 2} \\
& \leq C e^{\left(d_{0}-d\right)(((N+1) \pi) / b)}\left\|w^{N}\right\|_{1 / 2, \Gamma_{d_{0}}}\left\|u^{N}\right\|_{1 / 2, \Gamma_{E}} \leq C e^{\left(d_{0}-d\right)(((N+1) \pi) / b)}\left\|u^{N}\right\|_{1, \Omega_{W}}^{2} .
\end{aligned}
$$

From $d>d_{0}$, we have that $\left\{u^{N}\right\}$ is bounded in $V$. So, there exists a subsequence $\left\{u^{N_{n}}\right\}$, s.t. $u^{N_{n}} \rightarrow \bar{u} \in V$. Then, similar with Lemma 3,4 in [18], we obtain (43).

Then, we have the following convergence theorem.

Theorem 2. Let $u \in H^{2}\left(\Omega_{W}\right)$, and assumptions (38)-(40) be satisfied. Then,

$$
\lim _{h \longrightarrow 0, N \longrightarrow+\infty}\left\|u-u_{h}^{N}\right\|_{1, \Omega_{W}}=0 .
$$

Next, we deduce the error estimates. We suppose that the solution $u$ of problem (1) satisfies

$$
\left.u\right|_{\Omega_{W}} \in V \cap W^{k, 2+\varepsilon}\left(\Omega_{W}\right), \quad \varepsilon>0, k \geq 2 .
$$

For simplicity, we also define some notations as follows:

$$
\begin{gathered}
D(u ; u, v) \triangleq A(u ; u, v)+B(u ; u, v), \\
D_{N}\left(u^{N} ; u^{N}, v\right) \triangleq A\left(u^{N} ; u^{N}, v\right)+B_{N}\left(u^{N} ; u^{N}, v\right), \\
D_{N}\left(u_{h}^{N} ; u_{h}^{N}, v_{h}\right) \triangleq A\left(u_{h}^{N} ; u_{h}^{N}, v_{h}\right)+B_{N}\left(u_{h}^{N} ; u_{h}^{N}, v_{h}\right) .
\end{gathered}
$$

Then, problems (18), (32), and (36) can be replaced by some simple forms, respectively. Moreover, we introduce the following bilinear form $D^{\prime}(u ; \cdot, \cdot)$ and $D_{N}^{\prime}\left(u^{N} ; \cdot, \cdot\right)$

$$
\begin{aligned}
D^{\prime}(u ; v, z)= & \int_{\Omega_{W}} \frac{\partial a}{\partial s}(x, u) v \nabla u \cdot \nabla z \mathrm{~d} x+\int_{\Omega_{W}} a(x, u) \nabla v \cdot \nabla z \mathrm{~d} x \\
& +\int_{0}^{b} \int_{0}^{b} \frac{\partial \widetilde{a}}{\partial s}(u) v \frac{\partial u}{\partial x_{2}^{\prime}}\left(d, x_{2}^{\prime}\right) \frac{\partial z}{\partial x_{2}}\left(d, x_{2}\right) \sum_{n=1}^{+\infty} \frac{2}{n \pi} \sin \frac{n \pi x_{2}^{\prime}}{b} \sin \frac{n \pi x_{2}}{b} \mathrm{~d} x_{2}^{\prime} \mathrm{d} x_{2} \\
& +\int_{0}^{b} \int_{0}^{b} \widetilde{a}(u) \frac{\partial v}{\partial x_{2}^{\prime}}\left(d, x_{2}^{\prime}\right) \frac{\partial z}{\partial x_{2}}\left(d, x_{2}\right) \sum_{n=1}^{+\infty} \frac{2}{n \pi} \sin \frac{n \pi x_{2}^{\prime}}{b} \sin \frac{n \pi x_{2}}{b} \mathrm{~d} x_{2}^{\prime} \mathrm{d} x_{2}, \\
D_{N}^{\prime}\left(u^{N} ; v, z\right)= & \int_{\Omega_{W}} \frac{\partial a}{\partial s}\left(x, u^{N}\right) v \nabla u^{N} \cdot \nabla z \mathrm{~d} x+\int_{\Omega_{W}} a\left(x, u^{N}\right) \nabla v \cdot \nabla z \mathrm{~d} x \\
& +\int_{0}^{b} \int_{0}^{b} \frac{\partial \widetilde{a}}{\partial s}\left(u^{N}\right) v \frac{\partial u^{N}}{\partial x_{2}^{\prime}}\left(d, x_{2}^{\prime}\right) \frac{\partial z}{\partial x_{2}}\left(d, x_{2}\right) \sum_{n=1}^{N} \frac{2}{n \pi} \sin \frac{n \pi x_{2}^{\prime}}{b} \sin \frac{n \pi x_{2}}{b} \mathrm{~d} x_{2}^{\prime} \mathrm{d} x_{2} \\
& +\int_{0}^{b} \int_{0}^{b} \widetilde{a}\left(u^{N}\right) \frac{\partial v}{\partial x_{2}^{\prime}}\left(d, x_{2}^{\prime}\right) \frac{\partial z}{\partial x_{2}}\left(d, x_{2}\right) \sum_{n=1}^{N} \frac{2}{n \pi} \sin \frac{n \pi x_{2}^{\prime}}{b} \sin \frac{n \pi x_{2}}{b} \mathrm{~d} x_{2}^{\prime} \mathrm{d} x_{2} .
\end{aligned}
$$


Suppose $V^{\prime}$ is the dual space of $V$. By (2) and continuity of $(\partial a / \partial s)(\cdot, u(\cdot))$, we obtain that $D^{\prime}(u ; \cdot, \cdot)$ is bounded on $V \times V$. Then, there exists an operator $T: V \longrightarrow V^{\prime}$ such that

$$
(T v, z)=D^{\prime}(u ; v, z), \quad \forall v, z \in V .
$$

Similar to Lemma 2.2 in [19], we have the lemma as follows:

Lemma 4. The following inequality,

$$
(T v, v)+K\left(\|v\|_{0, \Omega_{W}}^{2}+\|v\|_{1 / 2, \Gamma_{E}}^{2}\right) \geq C\|v\|_{1, \Omega_{W}}^{2}, \quad \forall v \in V,
$$

holds, where $K \geq 0$ is a sufficient large constant.

We suppose that

$$
D^{\prime}(u ; v, z)=0, \quad \forall z \in V \Rightarrow v=0 .
$$

Assume $I: V \longrightarrow V^{\prime}$ is the canonical injection. Since $V$ is compactly embedded in $L^{2}\left(\Omega_{W}\right)$, we obtain that the operator $J: V \longrightarrow V^{\prime}$ defined by $J(v)=(I(v), 0)$ is also compact. Then, we deduce that $T: V \longrightarrow V^{\prime}$ is an isomorphism.
By conditions (19), (52), and (53) and Theorem 10.1.2 in [24], there exists $h_{0} \in(0,1]$, s.t. the following inf-sup condition is satisfied:

$$
\sup _{z \in V_{h}} \frac{D^{\prime}(u ; v, z)}{\|z\|_{1, \Omega_{W}}} \geq \alpha_{1}\|v\|_{1, \Omega_{W}}, \quad \forall v \in V_{h},
$$

where $\alpha_{1}>0$ is a constant independent of $h\left(h<h_{0}\right)$.

We define the Galerkin projection with respect to $D^{\prime}(u ; \cdot, \cdot)$ and $P_{h}: V \longrightarrow V_{h}$ :

$$
D^{\prime}\left(u ; P_{h} v, z\right)=D^{\prime}(u ; v, z), \quad \forall z \in V_{h} .
$$

Then, we obtain

$$
\left\|v-P_{h} v\right\|_{1, p, \Omega_{W}} \leq C \inf _{v_{h} \in V_{h}}\left\|v-v_{h}\right\|_{1, p, \Omega_{W}} \leq C h^{\sigma},
$$

where $2 \leq p \leq \infty, 0<\sigma<1$.

Lemma 5. $u_{h}^{N} \in V_{h}$ is a solution of (36) if and only if the following equation,

$$
D_{N}^{\prime}\left(u^{N} ; u^{N}-u_{h}^{N}, v\right)=R\left(u^{N} ; u_{h}^{N}, v\right), \quad \forall v \in V_{h},
$$

holds, where

$$
\begin{aligned}
& R\left(u^{N} ; u_{h}^{N}, v\right) \\
& \triangleq \int_{\Omega_{W}}\left(\int_{0}^{1}\left(\frac{\partial^{2} a}{\partial s^{2}}\left(x, w_{h}^{N}\right) \nabla w_{h}^{N} \cdot \nabla v\right)(1-t) \mathrm{d} t\right)\left(d_{h}^{N}\right)^{2} \mathrm{~d} x \\
& +2 \int_{\Omega_{W}}\left(\int_{0}^{1}\left(\frac{\partial a}{\partial s}\left(x, w_{h}^{N}\right) \nabla d_{h}^{N} \cdot \nabla v\right)(1-t) \mathrm{d} t\right) d_{h}^{N} \mathrm{~d} x \\
& +\int_{0}^{b} \int_{0}^{b}\left(\int_{0}^{1} \frac{\partial^{2} \widetilde{a}}{\partial s^{2}}\left(w_{h}^{N}\right) \frac{\partial w_{h}^{N}}{\partial x_{2}^{\prime}} \frac{\partial v}{\partial x_{2}} \sum_{n=1}^{N} \frac{2}{n \pi} \sin \frac{n \pi x_{2}^{\prime}}{b} \sin \frac{n \pi x_{2}}{b}\right)(1-t) \mathrm{d} t\left(d_{h}^{N}\right)^{2} \mathrm{~d} x_{2}^{\prime} \mathrm{d} x_{2} \\
& \quad+2 \int_{0}^{b} \int_{0}^{b}\left(\int_{0}^{1}\left(\frac{\partial \widetilde{a}}{\partial s}\left(w_{h}^{N}\right) \frac{\partial d_{h}^{N}}{\partial x_{2}^{\prime}} \frac{\partial v}{\partial x_{2}} \sum_{n=1}^{N} \frac{2}{n \pi} \sin \frac{n \pi x_{2}^{\prime}}{b} \sin \frac{n \pi x_{2}}{b}\right)(1-t) \mathrm{d} t\right) d_{h}^{N} \mathrm{~d} x_{2}^{\prime} \mathrm{d} x_{2},
\end{aligned}
$$

with $w_{h}^{N}=u^{N}+t\left(u_{h}^{N}-u^{N}\right), d_{h}^{N}=u_{h}^{N}-u^{N}$.

Proof. Suppose $\eta(t) \triangleq D_{N}\left(w_{h}^{N} ; w_{h}^{N}, v\right)$. Then, by (32) and (36) and

$$
\eta(1)=\eta(0)+\eta^{\prime}(0)+\int_{0}^{1} \eta^{\prime}(t)(1-t) \mathrm{d} t .
$$

we can obtain the desired result.

Suppose

$$
M_{h} \triangleq\left\{v \in V_{h} \mid\|v\|_{1, \infty, \Omega_{W}} \leq 1+\left\|u^{N}\right\|_{1, \infty, \Omega_{W}}\right\} .
$$

Then, from $[19,25]$, we have the following lemma.

Lemma 6. There exists a constant $C>0$ independent of $h$, such that

$$
\begin{aligned}
& \left|R\left(u^{N} ; v, z\right)\right| \leq C\left(\left\|u^{N}-v\right\|_{1, \Omega_{W}}^{2}+\left\|u^{N}-v\right\|_{1, \Omega_{W}}\right)\|z\|_{1, \Omega_{W}}, \\
& \quad \forall v \in M_{h}, \quad \forall z \in V_{h} .
\end{aligned}
$$

We denote a nonlinear mapping $\psi: V_{h} \longrightarrow V_{h}$, which satisfies that $\psi(v)$ is the unique solution of

$$
D^{\prime}(u ; \psi(v), z)=D^{\prime}(u ; u, z)-R(u ; v, z), \quad \forall z \in V_{h} \text {, }
$$

for any given $v \in V_{h}$. Suppose

$$
E_{h} \triangleq\left\{v \in V_{h} \mid\left\|v-P_{h} v\right\|_{1, \infty, \Omega_{W}} \leq C h^{\sigma}\right\} .
$$

Then, we obtain the lemma as follows. 
Lemma 7. The nonlinear mapping $\psi$ is a continuous mapping from $E_{h}$ to $E_{h}$.

Proof. By (62), we have

$$
D^{\prime}\left(u ; \psi(v)-\psi\left(v_{n}\right), z\right)=R\left(u ; v_{n}, z\right)-R(u ; v, z) .
$$

Combining (64) with (54), we deduce that the mapping $\psi$ is continuous, i.e.,

$$
\lim _{v_{n} \longrightarrow v} \psi\left(v_{n}\right)=\psi(v)
$$

For any $v \in E_{h}$,

$$
\begin{aligned}
& \|v\|_{1, \infty, \Omega_{W}} \leq\left\|u^{N}-v\right\|_{1, \infty, \Omega_{W}}+\left\|u^{N}\right\|_{1, \infty, \Omega_{W}}, \\
& \left\|u^{N}-v\right\|_{1, \infty, \Omega_{W}} \leq\left\|u^{N}-P_{h} u^{N}\right\|_{1, \infty, \Omega_{W}}+\left\|P_{h} u^{N}-v\right\|_{1, \infty, \Omega_{W}},
\end{aligned}
$$

$$
\begin{aligned}
\left\|u^{N}-P_{h} u^{N}\right\|_{1, \infty, \Omega_{W}} \leq & \left\|u^{N}-\Pi_{h} u^{N}\right\|_{1, \infty, \Omega_{W}} \\
& +\left\|\Pi_{h} u^{N}-P_{h} u^{N}\right\|_{1, \infty, \Omega_{W}} .
\end{aligned}
$$

Since $\mathscr{J}_{h}$ is regular and quasi-uniform, according to [26], we have the following inverse inequality:

$$
\|w\|_{1, \infty, \Omega_{W}} \leq C\left(\log \frac{1}{h}\right)^{1 / 2}\|w\|_{1, \Omega_{W}}, \quad \forall w \in V_{h} .
$$

By the definition of $E_{h}$, (56), and (69), we obtain

$$
\left\|u^{N}-v\right\|_{1, \infty, \Omega_{W}} \leq 1
$$

This implies that $v \in M_{h}$. Under the definition of $P_{h}$, (62) can be rewritten as

$$
D^{\prime}\left(u^{N} ; \psi(v)-P_{h} u^{N}, z\right)=-R\left(u^{N} ; v, z\right), \quad \forall z \in V_{h} .
$$

Then, by (54) and Lemmas 5 and 6, we obtain

$$
\begin{aligned}
\left\|\psi(v)-P_{h} u^{N}\right\|_{1, \Omega_{W}} & \leq C \sup _{z \in V_{h}} \frac{\left|D^{\prime}\left(u ; \psi(v)-P_{h} u^{N}, z\right)\right|}{\|z\|_{1, \Omega_{W}}} \\
& \leq C\left(\left\|u^{N}-v\right\|_{1, \Omega_{W}}^{2}+\left\|u^{N}-v\right\|_{1, \Omega_{W}}\right) \\
& \leq C\left(\left\|u^{N}-P_{h} u^{N}\right\|_{1, \Omega_{W}}^{2}+\left\|P_{h} u^{N}-v\right\|_{1, \Omega_{W}}^{2}+\left\|u^{N}-P_{h} u^{N}\right\|_{1, \Omega_{W}}+\left\|P_{h} u^{N}-v\right\|_{1, \Omega_{W}}\right) \\
& \leq C h^{\sigma} .
\end{aligned}
$$

This means that $\psi: E_{h} \longrightarrow E_{h}$.

Theorem 3. Suppose $u \in V \cap W^{k, 2+\varepsilon}\left(\Omega_{W}\right)$ is a solution of problem (1), where $\varepsilon>0, k \geq 2$. We also assume that
$\left.u\right|_{\Gamma_{d_{0}}} \in H^{k-(1 / 2)}\left(\Gamma_{d_{0}}\right)$ and $u$ satisfies (53). With sufficiently small $h$, problem (36) has an approximate solution $u_{h}^{N} \in V_{h}$, such that

$$
\left\|u-u_{h}^{N}\right\|_{1, \Omega_{W}} \leq C\left(h^{\sigma}+\frac{1}{(N+1)^{k-1}} e^{\left(d_{0}-d\right)(((N+1) \pi) / b)}\|u\|_{k-\frac{1}{2}, \Gamma_{d_{0}}}\right) .
$$

Proof. By Brouwer's fixed-point theorem and Lemma 7, $\left\|u^{N}-u_{h}^{N}\right\|_{1, \Omega_{W}} \leq\left\|u^{N}-P_{h} u^{N}\right\|_{1, \Omega_{W}}+\left\|P_{h} u^{N}-u_{h}^{N}\right\|_{1, \Omega_{W}} \leq C h^{\sigma}$, there exists $u_{h}^{N} \in V_{h}$, such that $\psi\left(u_{h}^{N}\right)=u_{h}^{N}$. From Lemma 5, we deduce that $u_{h}^{N}$ is a solution of (36). Moreover, by (56) and $u_{h}^{N} \in E_{h}$, we have

For any $u^{N} \in V$, according to Lemma 3, we obtain 


$$
\begin{aligned}
& \left|B\left(u^{N} ; u^{N}, v\right)-B_{N}\left(u^{N} ; u^{N}, v\right)\right| \\
& \leq C e^{\left(d_{0}-d\right)(((N+1) \pi) / b)}\left(\sum_{n=N+1}^{+\infty}\left(1+n^{2}\right)^{1 / 2} a_{n}^{2}\right)^{1 / 2}\left(\sum_{n=N+1}^{+\infty}\left(1+n^{2}\right)^{1 / 2} c_{n}^{2}\right)^{1 / 2} \\
& \leq C \frac{1}{(N+1)^{k-1}} e^{\left(d_{0}-d\right)(((N+1) \pi) / b)}\left(\sum_{n=N+1}^{+\infty}\left(1+n^{2}\right)^{k-1 / 2} a_{n}^{2}\right)^{1 / 2}\left(\sum_{n=N+1}^{+\infty}\left(1+n^{2}\right)^{1 / 2} c_{n}^{2}\right)^{1 / 2} \\
& \leq C \frac{1}{(N+1)^{k-1}} e^{\left(d_{0}-d\right)(((N+1) \pi) / b)}\|u\|_{k-(1 / 2), \Gamma_{d_{0}}} v_{1, \Omega_{W} .}
\end{aligned}
$$

From (32), we have

$$
\begin{aligned}
D\left(u^{N} ; u^{N}, v\right) & =A\left(u^{N} ; u^{N}, v\right)+B\left(u^{N} ; u^{N}, v\right) \\
& =F(v)+B\left(u^{N} ; u^{N}, v\right)-B_{N}\left(u^{N} ; u^{N}, v\right) .
\end{aligned}
$$

Let $\eta(t)=D\left(u+t\left(u^{N}-u\right) ; u+t\left(u^{N}-u\right), v\right)$; then,

$$
\int_{0}^{1} D^{\prime}\left(u+t\left(u^{N}-u\right) ; u^{N}-u, v\right) \mathrm{d} t=D\left(u^{N} ; u^{N}, v\right)-D(u ; u, v) .
$$

By (18), (52), and (53) and [24], we have

$$
\begin{aligned}
\left\|u-u^{N}\right\|_{1, \Omega_{W}} & \leq C \sup _{v \in V} \frac{\int_{0}^{1} D^{\prime}\left(u+t\left(u^{N}-u\right) ; u^{N}-u, v\right) d t}{\|v\|_{1, \Omega_{W}}} \\
& \leq C \frac{\left|B\left(u^{N} ; u^{N}, v\right)-B_{N}\left(u^{N} ; u^{N}, v\right)\right|}{\|v\|_{1, \Omega_{W}}} \leq C \frac{1}{(N+1)^{k-1}} e^{\left(d_{0}-d\right)(((N+1) \pi) / b)}\|u\|_{k-(1 / 2), \Gamma_{d_{0}}} .
\end{aligned}
$$

Combining (74) with (78), we obtain

$$
\begin{aligned}
\left\|u-u_{h}^{N}\right\|_{1, \Omega_{W}} & \leq\left\|u-u^{N}\right\|_{1, \Omega_{W}}+\left\|u^{N}-u_{h}^{N}\right\|_{1, \Omega_{W}} \\
& \leq C\left(h^{\sigma}+\frac{1}{(N+1)^{k-1}} e^{\left(d_{0}-d\right)(((N+1) \pi) / b)}\|u\|_{k-(1 / 2), \Gamma_{d_{0}}}\right) .
\end{aligned}
$$

This completes the proof.

\section{Numerical Examples}

In this section, we computed some numerical examples by the method developed in Sections 2 and 3 to test the efficiency of the method.

Example 1. We take $\Omega=\left\{\left(x_{1}, x_{2}\right) \mid x_{1}>0, \quad 0<x_{2}<b\right\}$, $\Gamma_{W}=\left\{\left(0, x_{2}\right) \mid 0<x_{2}<b\right\}, \Gamma_{N}=\left\{\left(x_{1}, b\right) \mid x_{1}>0\right\}, \Gamma_{S}=\left\{\left(x_{1}, 0\right) \mid\right.$ $\left.x_{1}>0\right\}, b=1$, and $a(x, u)=\left(1 /\left(1+u^{2}\right)\right)$. The exact solution of original problem is $u=\tan \left(\sum_{m=1}^{3}\left(1 / m^{2}\right) e^{-\left(m \pi x_{1} / b\right)}\right.$ $\left.\cos \left(m \pi x_{2} / b\right)\right)$. Let $\Gamma_{E}=\left\{\left(x_{1}, x_{2}\right) \mid x_{1}=d, \quad 0<x_{2}<b\right\}$ be the artificial boundaries. Figure 3 shows the mesh $h$ of subdomain $\Omega_{W}(d=1)$. The numerical results are given in Table 1 and Figures 4 and 5.

From the numerical results, we can deduce that the finite element mesh, the location of artificial boundary, and the truncation terms of series can affect the numerical errors. It is obvious that our method is very effective. 


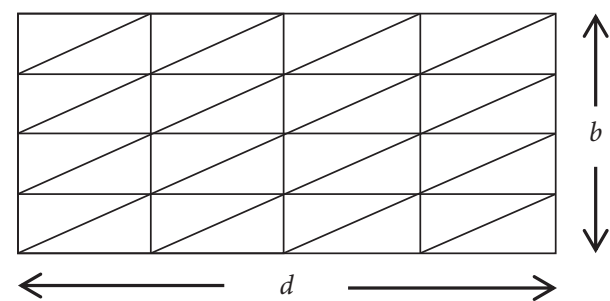

Figure 3: Mesh $h$ of subdomain $\Omega_{W}$.

Table 1: The errors with different meshes $(d=1, N=20)$.

\begin{tabular}{lcc}
\hline Mesh & $L^{2}\left(\Omega_{W}\right)$ error & $L^{\infty}\left(\Omega_{W}\right)$ error \\
\hline$h$ & 0.027864 & 0.059187 \\
$h / 2$ & 0.007744 & 0.026333 \\
$h / 4$ & 0.002033 & 0.009699 \\
$h / 8$ & 0.000504 & 0.002785 \\
\hline
\end{tabular}

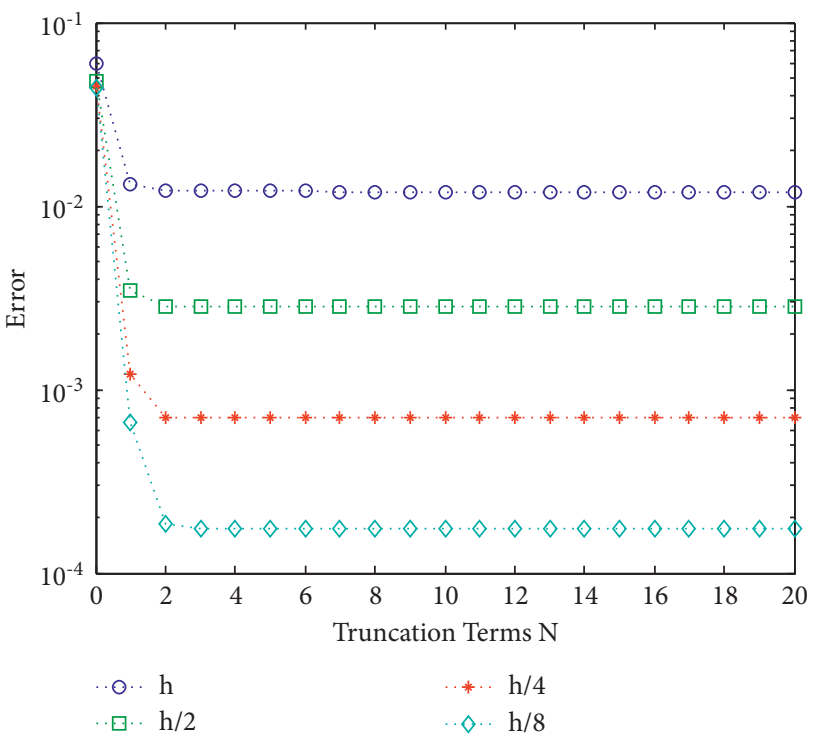

Figure 4: $L^{\infty}\left(\Gamma_{E}\right)$ errors with different $N(d=1)$.

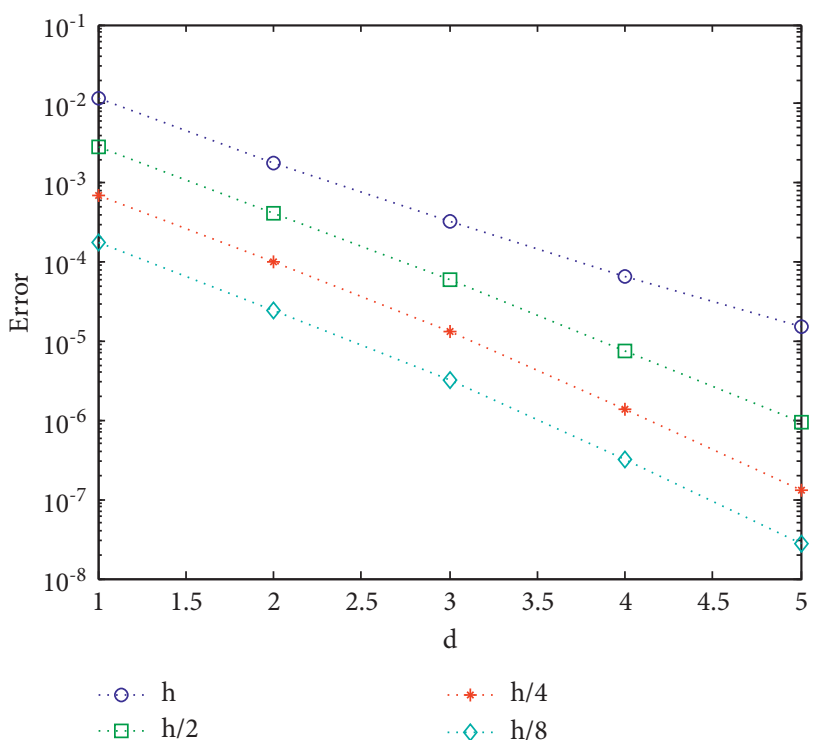

Figure 5: $L^{\infty}\left(\Gamma_{E}\right)$ errors with different $d(N=20)$.

\section{Conclusions}

In this paper, we propose a method of artificial boundary conditions for quasi-linear problems in semi-infinite strips by using a segment artificial boundary. The exact and approximate artificial boundary conditions are given based on the Kirchhoff transformation. A new error estimate for the finite element approximation with the approximate artificial boundary condition is obtained. Finally, some numerical examples show the efficiency of this method. The quasilinear problem, we considered in this paper, is a two-dimensional problem. Based on the proposed method, one can design some artificial boundary conditions for three-dimensional problem; we shall report on progress in some of these directions in a future publication.

\section{Data Availability}

No data were used to support this study.

\section{Conflicts of Interest}

The authors declare that they have no conflicts of interest.

\section{Acknowledgments}

This research was funded by the National Natural Science Foundation of China (no. 11371198).

\section{References}

[1] I. Hlavacek, M. Krizek, and J. Maly, "On Galerkin approximations of a quasilinear nonpotential elliptic problem of a nonmonotone type," Journal of Mathematical Analysis and Applications, vol. 184, no. 1, pp. 168-189, 1994.

[2] I. Hlavacek, "A note on the Neumann problem for a quasilinear elliptic problem of a nonmonotone type," Journal of Mathematical Analysis and Applications, vol. 211, no. 1, pp. 365-369, 1997.

[3] S.-S. Chow, "Finite element error estimates for non-linear elliptic equations of monotone type," Numerische Mathematik, vol. 54, no. 4, pp. 373-393, 1989.

[4] S. Korotov and M. Kř́žžek, "Finite element analysis of varitional crimes for a quasilinear elliptic problem in 3D," Numerische Mathematik, vol. 84, no. 4, pp. 549-576, 2000.

[5] F. A. Milner, "Mixed finite element methods for quasilinear second-order elliptic problems," Mathematics of Computation, vol. 44, no. 170, p. 303, 1985.

[6] S. Meddahi, "On a mixed finite element formulation of a second-order quasilinear problem in the plane," Numerical Methods for Partial Differential Equations, vol. 20, no. 1, pp. 90-103, 2004.

[7] M. Karchevsky and A. Fedotov, "Error estimates and iterative procedure for mixed finite element solution of second-order quasi-linear elliptic problems," Computational Methods in Applied Mathematics, vol. 4, no. 4, pp. 445-463, 2004.

[8] T. Wongcharoen, B. M. A. Rahman, and K. T. V. Grattan, "Finite element analysis of optical directional couplers," ScienceAsia, vol. 21, no. 3, pp. 161-174, 1995.

[9] P. Houston, J. Robson, and E. Süli, "Discontinuous Galerkin finite element approximation of quasilinear elliptic boundary 
value problems I: the scalar case," IMA Journal of Numerical Analysis, vol. 25, no. 4, pp. 726-749, 2005.

[10] E. M. Garau, P. Morin, and C. Zuppa, "Convergence of an adaptive Kačanov FEM for quasi-linear problems," Applied Numerical Mathematics, vol. 61, no. 4, pp. 512-529, 2011.

[11] $\mathrm{H}$. Han and $\mathrm{X}$. Wu, "Approximation of infinite boundary condition and its application to finite element methods," Journal of Computational Mathematics, vol. 3, no. 2, pp. 179-192, 1985.

[12] H. Han and X. Wu, The Artificial Boundary Method - Numerical Solutions of Partial Differential Equations on Unbounded Domains, Tsinghua University Press, Beijing, China, 2009.

[13] K. Feng, "Finite element method and natural boundary reduction," in Proceedings of International Congress Mathematicians, Warsaw, Poland, August 1983.

[14] K. Feng and D. Yu, "Canonical integral equations of elliptic boundary value problems and their numerical solutions," in Proceedings of China-France Symposium on the Finite Element Methods, Monterey, CA, USA, 1983.

[15] D. Yu, Natural Boundary Integral Method and its Applications, Kluwer Academic Publishers, Amsterdam, Netherlands, 2002.

[16] J. B. Keller and D. Givoli, "Exact non-reflecting boundary conditions," Journal of Computational Physics, vol. 82, no. 1, pp. 172-192, 1989.

[17] M. J. Grote and J. B. Keller, "On nonreflecting boundary conditions," Journal of Computational Physics, vol. 122, no. 2, pp. 231-243, 1995.

[18] H. Han, Z. Huang, and D. Yin, "Exact artificial boundary conditions for quasilinear elliptic equations in unbounded domains," Communications in Mathematical Sciences, vol. 6, no. 1, pp. 71-82, 2008.

[19] D. Liu and D. Yu, "A FEM-BEM formulation for an exterior quasilinear elliptic problem in the plane," Journal of Computational Mathematics, vol. 26, no. 3, pp. 378-389, 2008.

[20] B. Liu and Q. Du, "On the coupled of NBEM and FEM for an anisotropic quasilinear problem in elongated domains," American Journal of Computational Mathematics, vol. 2, no. 2, pp. 143-155, 2012.

[21] B. Liu and Q. Du, "The coupling of NBEM and FEM for quasilinear problems in a bounded or unbounded domain with a concave angle," Journal of Computational Mathematics, vol. 31, no. 3, pp. 308-325, 2013.

[22] D. B. Ingham and M. A. Kelmanson, Boundary Integral Equation Analyses of Singular, Potential, and Biharmonic Problems, Springer-Verlag, Berlin, Germany, 1984.

[23] Y. Chen and Q. Du, "Artificial boundary method for anisotropic problems in semi-infinite strips," Engineering Letters, vol. 26, no. 4, pp. 473-477, 2018.

[24] C. Chen and J. Zhou, Boundary Element Methods, Academic Press, London, UK, 1992.

[25] S. Meddahi, M. Gonzalez, and P. Perez, "On a FEM--BEM formulation for an exterior quasilinear problem in the plane," SIAM Journal on Numerical Analysis, vol. 37, no. 6, pp. 1820-1837, 2000.

[26] J. Xu, "Theory of Multilevel Methods," Ph.D. Thesis, Cornell University, NY, USA, 1989. 